\title{
Boron application influenced growth, yield and fiber quality of upland cotton (Gossipium hirsutum L.) under different irrigation regimes
}

\author{
Ayaz Ahmad ${ }^{1 *}$, Waseem Hassan ${ }^{1}$, Hakoomat Ali ${ }^{1}$, Muhammad Irfan ${ }^{1}$ \\ and Riaz Ahmad ${ }^{2}$ \\ 1. Department of Agronomy, Bahauddin Zakariya University, 60800 Multan-Pakistan \\ 2. Department of Horticulture, Bahauddin Zakariya University, 60800 Multan-Pakistan \\ *Corresponding author's email: ayazagronomist@gmail.com \\ Citation \\ Ayaz Ahmad, Waseem Hassan, Hakoomat Ali, Muhammad Irfan and Riaz Ahmad. Boron application influenced \\ growth, yield and fiber quality of upland cotton (Gossipium hirsutum L.) under different irrigation regimes. Pure \\ and Applied Biology. Vol. 10, Issue 4, pp1345-1355. http://dx.doi.org/10.19045/bspab.2021.100139
}

\begin{tabular}{llll}
\hline \hline Received: 12/12/2020 & Revised: 19/02/2021 & Accepted: 26/02/2021 & Online First: 25/03/2021 \\
\hline \hline
\end{tabular}

\section{Abstract}

Cotton is a major imperative cash crop crop globally including Pakistan. Among abiotic stresses, drought stress is critical that suppress plant growth, reduce crop production, low fiber quality and poor earnings for farmers. Nutrient deficiency is major factor resulting in poor crop production. Sufficient nutrient application has better capability to increase yield because it helps to plants to stand against adverse climatic conditions within the growing areas. Therefore, present study was conducted on evaluation of cultivars performance applying numerous irrigation systems and use of boron through different methods. Cultivar CIM-678 had maximum growth traits, allometry parameters and fiber quality attributes than CIM-602 which had minimum growth, allometry and fiber quality traits. Foliar application of boron and field capacity (75\%) also increased plant growth, allometry and fiber traits. The interactive effects of cultivars, boron use and field capacity were improved the plant growth, yield, allometry parameter and fiber quality traits. Conclusively, CIM-678, boron foliar spray and field capacity (75\%) improved the cotton crop production with excellent fiber quality traits.

Keywords: BCR; Drought stress; Field capacity; Foliar spray

\section{Introduction}

Cotton is more popular as "white gold" because of higher economic potential in tropical and subtropical regions including Pakistan. Generally, textile industry mainly based on natural fiber for different mankind purposes [1]. Among oil seed crop, cotton is one of the major imperative oil producing crop globally including Pakistan [2]. Recently, cotton production faced different problems like poor soils, improper nutrition management practices, insufficient irrigations, much weeds competition, bombardment of different hazards chemicals and selection of low yielding cultivars and abiotic stresses resulting in poor crop growth with low fiber quality related traits $[3,4]$.

Water stress conditions are more critical to suppress plant growth, reduce crop production, low fiber quality and poor earnings for farmers [5]. Drought stress conditions reduced plant size, flowering, bolls set per plant, boll size, fiber elongation, strength, length, uniformity as well as micronair [6]. Soil is found to be 
very poor because of use of brackish water or water shortage [7]. Different morphological, physiological and biochemical properties were disturbed because of drought stress conditions $[6,8]$. Photosynthetic imbalance, disturbance of electrolyte leakage, respiration impairing, poor accumulation of carbohydrates, imbalance ion exchange, cell division restriction and nutritional metabolism [9, 10]. Consequently, it is more urgent and time need to situate more efforts aimed at improvement of cotton with excellent traits by using several drought resistant cultivars, better management practices, sufficient nutrition management and numerous cultural practices.

Numerous physiological changes were occurred in plants under drought stress. Nutrient deficiency in plants may possibly be occurred because of soil compaction, excess fertilization and intensive cropping systems $[11,12]$. Exogenous application of different macronutrients and micronutrients had better potential to increase crop production. Moreover, these have potential to increase enhance plants defense mechanism against different biotic and abiotic stresses especially against drought stress [1]. Deficiency of different micronutrients such as manganese, copper, sulfur, boron and zinc is found to be more common in agronomic crops in developing countries [13]. Many improvements in nutrition management are necessary for increase of cotton production with good quality fiber traits [14]. Several earlier research work proved that application of different nutrients especially boron increased plant defense mechanism and reduce drought stress conditions resulting in better performance of cotton yield [5].

The cultivars performance under water stress varied from one cultivars to other cultivar because of different genetic background [15]. Boron is significantly involved in morpho-physical and biochemical variation especially translocation of sugars, formation of meristem tissues, photosynthetic mechanism, cell division, protein synthesis and metabolism of nutrients. Boron may have potential to scavenge ROS by increasing oxidative as well as nonoxidative scavengers in plants [7]. Sufficient nutrient application has better capability to increase yield because it helps to plants to stand against adverse climatic conditions. The identification and evaluation of drought resistant cultivars and their important is very important goal for plant researchers. It my assumed that boron had potential increase the defense mechanism of plants under drought stress. Current experiment was aimed to evaluate the effect of boron application on different cotton cultivars regarding their growth, allometry and fiber traits.

\section{Materials and Methods}

Current study was conducted and analyzed at the Central Cotton Research Institute (CCRI) Multan. Current experiment was arranged under randomized Complete Block (RCBD) having factorial arrangement with four repeats. Plot size was $5 \mathrm{~m} \times 10 \mathrm{~m}$ and seeds were sown on $26^{\text {th }}$ May, 2017 and on the $28^{\text {th }}$ May, 2018. Pickings were start at 04 -11-2017 and 0711-2018. Several pickings were harvested after one week interval.

\section{Treatments}

Two cultivars i.e. CIM-678 and CIM-602 were studied. Boron was applied through different methods i.e. applied in soil $\left(B_{1}\right)$, treated the seeds $\left(\mathrm{B}_{2}\right)$ and foliar spray $\left(\mathrm{B}_{3}\right)$. Moreover, three irrigation regimes i.e. $75 \%$ field capacity $\left(\mathrm{I}_{1}\right), 50 \%$ field capacity $\left(\mathrm{I}_{2}\right)$ and $25 \%$ field capacity $\left(\mathrm{I}_{3}\right)$.

\section{Observations}

\section{Agronomic traits}

Twelve plants were used from each treatment for estimation of plant height, number of bolls/ plant, boll weight, seed cotton, lint and biological yield, and seed cotton harvest in the current study.

\section{Allometry traits}

Leaf area index $=\frac{\text { Leaf area }}{\text { Ground area leaf }}$

Leaf area meter (CI-203, BioScientific, USA) was used to measure leaf area of 
crop.

Leaf area duration $=\frac{(\mathrm{LAI} 1+\mathrm{LAI} 2) \times(\mathrm{T} 2-\mathrm{T} 1)}{2}$

Leaf area duration was calculated by $\mathrm{LAI}_{1}$ and $\mathrm{LAI}_{2}$ with leaf area indices at times $\mathrm{T}_{1}$ and $\mathrm{T}_{2}$ and $\mathrm{LAD}$ (days) $=\left(\mathrm{LAI}_{1}+\mathrm{LAI}_{2}\right) \times$ $\left(\mathrm{T}_{2}-\mathrm{T}_{1}\right) / 2[1]$.

Crop growth rate was calculated from weighed samples of 10, 30, $60 \mathrm{~g}$ of green leaves, stalks and bolls collected from all the studied treatments and samples were dried at $80{ }^{\circ} \mathrm{C}$. Net assimilation rate was determined from formula as determined [1]. Fiber quality traits

Fiber traits such as fiber length, elongation, uniformity, strength and micronair were calculated using High Volume Instrument (HVI Spectrum).

\section{Data analyses}

The collected data were separated using Statistix 8.1 software through analysis of variance technique under three way factorial [12]. LSD test was used for means separation under 5\% probability [10].

Results

Among growth traits, cultivar CIM-678 had longer plant height $(164.74 \mathrm{~cm})$ than CIM$602(158.81 \mathrm{~cm})$. Among boron application, foliar application showed higher plant height $(165.89 \mathrm{~cm})$ than other studied boron application methods. Among irrigation regimes, field capacity (75\%) showed taller plant height $(164.11 \mathrm{~cm})$ than field capacity $(25 \%)(159.22 \mathrm{~cm})$ (Table 1$)$. The interactive effect of cultivars, boron use as well as irrigation regimes for plant height were presented in the (Fig. 1). CIM602 had higher bolls number/ plant (38.78) as compared CIM-678 (33.44). Foliar spray of boron showed highest bolls number/ plant (38.22) than other studied boron application methods. Among irrigation regimes, field capacity $(75 \%)$ showed greater number of bolls/ plant (38.33) than field capacity (25\%) (33.50). The interaction of cultivars, boron application as well as irrigation systems for bolls number/ plant (Fig. 1). CIM-602 had maximum boll weight $(1.54 \mathrm{~g})$ than CIM678 (1.27 g). Application of boron on leaf showed higher boll weight $(1.54 \mathrm{~g})$ than other studied boron application methods. Among irrigation regimes, field capacity $(75 \%)$ showed greater boll weight $(1.60 \mathrm{~g})$ than field capacity $(25 \%)(1.12 \mathrm{~g})$. The interface of cultivars, boron use and irrigations for boll weight were presented (Fig. 1).

Table 1. Growth related traits as affected from cultivars, boron application and field capacity

\begin{tabular}{|c|c|c|c|c|c|c|}
\hline Treatments & $\begin{array}{c}\text { Plant } \\
\text { height } \\
(\mathbf{c m})\end{array}$ & $\begin{array}{c}\text { Number of } \\
\text { bolls/plant }\end{array}$ & $\begin{array}{c}\text { Boll } \\
\text { weight } \\
(\mathbf{g})\end{array}$ & $\begin{array}{c}\text { Seed cotton } \\
\text { yield (Kg ha }\end{array}$ & $\begin{array}{c}\text { Lint yield } \\
\mathbf{( K g ~ h a}\end{array}$ & $\begin{array}{c}\text { Seed cotton } \\
\text { harvest } \\
(\boldsymbol{\%})\end{array}$ \\
\hline CIM-678 & $158.81 \mathrm{~B}$ & $33.44 \mathrm{~B}$ & $1.27 \mathrm{~B}$ & $2078.1 \mathrm{~B}$ & $31.29 \mathrm{~B}$ & $18.89 \mathrm{~B}$ \\
\hline CIM-602 & $164.74 \mathrm{~A}$ & $38.78 \mathrm{~A}$ & $1.54 \mathrm{~A}$ & $2252.2 \mathrm{~A}$ & $37.59 \mathrm{~A}$ & $24.18 \mathrm{~A}$ \\
\hline Soil application & $163.33 \mathrm{C}$ & $35.56 \mathrm{C}$ & $1.29 \mathrm{C}$ & $2164.3 \mathrm{~B}$ & $32.78 \mathrm{C}$ & $20.00 \mathrm{C}$ \\
\hline Seed treatment & $165.00 \mathrm{~B}$ & $37.67 \mathrm{~B}$ & $1.46 \mathrm{~B}$ & $2165.3 \mathrm{~A}$ & $35.00 \mathrm{~B}$ & $21.83 \mathrm{~B}$ \\
\hline $\begin{array}{c}\text { Foliar } \\
\text { application }\end{array}$ & $165.89 \mathrm{~A}$ & $38.22 \mathrm{~A}$ & $1.47 \mathrm{~A}$ & $2165.8 \mathrm{~A}$ & $35.56 \mathrm{~A}$ & $22.72 \mathrm{~A}$ \\
\hline $\begin{array}{c}\text { Field capacity } \\
(25 \%)\end{array}$ & $159.22 \mathrm{C}$ & $33.50 \mathrm{C}$ & $1.12 \mathrm{C}$ & $2029.8 \mathrm{C}$ & $31.83 \mathrm{C}$ & $19.00 \mathrm{C}$ \\
\hline $\begin{array}{c}\text { Field capacity } \\
(50 \%)\end{array}$ & $162.00 \mathrm{~B}$ & $36.50 \mathrm{~B}$ & $1.49 \mathrm{~B}$ & $2182.0 \mathrm{~B}$ & $34.78 \mathrm{~B}$ & $21.89 \mathrm{~B}$ \\
\hline $\begin{array}{c}\text { Field capacity } \\
(75 \%)\end{array}$ & $164.11 \mathrm{~A}$ & $38.33 \mathrm{~A}$ & $1.60 \mathrm{~A}$ & $2283.7 \mathrm{~A}$ & $36.72 \mathrm{~A}$ & $23.67 \mathrm{~A}$ \\
\hline
\end{tabular}






Figure 1. Plant height, number of bolls/plant, boll weight as affected from cultivars, boron application and field capacity

CIM-602 had higher seed cotton yield (2252.2 $\left.\mathrm{Kg} \mathrm{ha}^{-1}\right)$, lint yield $\left(37.59 \mathrm{Kg} \mathrm{ha}^{-1}\right)$ and seed cotton harvest $(24.18 \%)$ than CIM-678. Application of boron on leaf showed maximum seed cotton yield $\left(2165.8 \mathrm{Kg} \mathrm{ha}^{-1}\right)$, lint yield (35.56 $\left.\mathrm{Kg} \mathrm{ha}^{-1}\right)$ and seed cotton harvest $(22.72 \%)$ than other studied boron application methods. Among irrigation regimes, field capacity $(75 \%)$ showed greater higher seed cotton yield $\left(2283.7 \mathrm{Kg} \mathrm{ha}^{-1}\right)$, lint yield (36.72 $\left.\mathrm{Kg} \mathrm{ha}^{-1}\right)$ and seed cotton harvest $(23.67 \%)$ than field capacity $(25 \%)$ (Table 2$)$. The interface of cultivars, boron use and numerous irrigations for seed cotton yield, lint yield and seed cotton harvest presented (Fig. 2). 
Table 2. Allometry traits affected from cultivars, boron application and field capacity

\begin{tabular}{|c|c|c|c|}
\hline Treatments & Leaf area index & $\begin{array}{c}\text { Leaf area duration } \\
(\mathbf{d a y s})\end{array}$ & $\begin{array}{c}\text { Net assimilation rate }(\mathbf{g} \\
\left.\mathbf{m}^{-\mathbf{2}} \mathbf{d a y}^{\mathbf{1}}\right)\end{array}$ \\
\hline CIM-678 & $0.34 \mathrm{~B}$ & $468.22 \mathrm{~B}$ & $1.03 \mathrm{~B}$ \\
\hline CIM-602 & $0.45 \mathrm{~A}$ & $587.26 \mathrm{~A}$ & $1.36 \mathrm{~A}$ \\
\hline Soil application & $0.38 \mathrm{C}$ & $526.33 \mathrm{~B}$ & $1.19 \mathrm{C}$ \\
\hline Seed treatment & $0.39 \mathrm{~B}$ & $528.50 \mathrm{~A}$ & $1.20 \mathrm{~B}$ \\
\hline Foliar application & $0.41 \mathrm{~A}$ & $528.39 \mathrm{~A}$ & $1.21 \mathrm{~A}$ \\
\hline Field capacity (25\%) & $0.36 \mathrm{C}$ & $450.11 \mathrm{C}$ & $1.15 \mathrm{C}$ \\
\hline Field capacity (50\%) & $0.40 \mathrm{~B}$ & $553.83 \mathrm{~B}$ & $1.20 \mathrm{~B}$ \\
\hline Field capacity (75\%) & $0.42 \mathrm{~A}$ & $579.28 \mathrm{~A}$ & $1.22 \mathrm{~A}$ \\
\hline
\end{tabular}

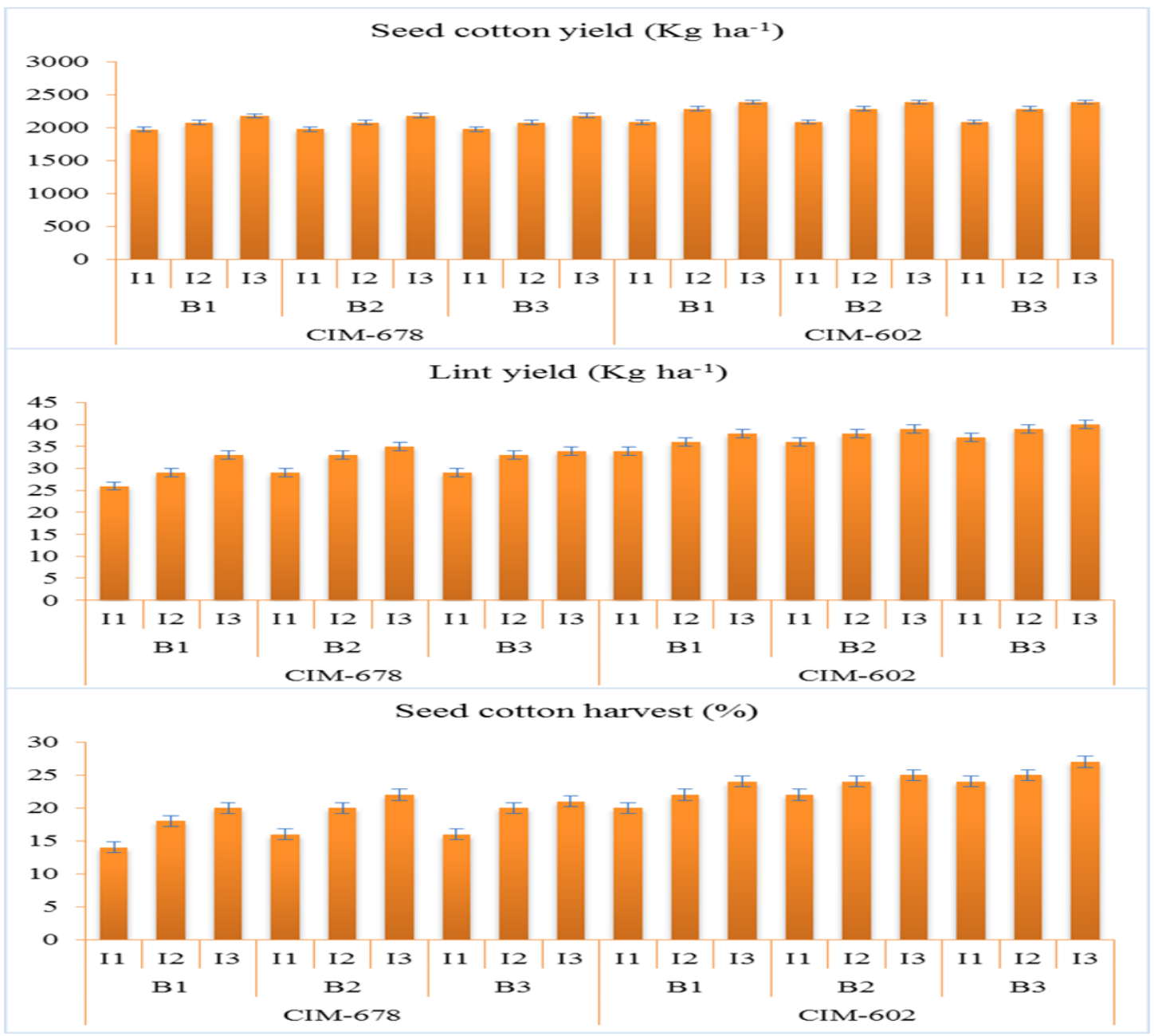

Figure 2. Seed cotton yield, lint yield and seed cotton harvest as affected from cultivars, boron application and field capacity

Regarding allometry traits, CIM-602 had greater leaf area index (0.45) than CIM-678 (0.34). Foliar application of boron showed higher leaf area index (0.41) than other studied boron application methods. Among irrigation regimes, field capacity $(75 \%)$ showed greater leaf area index (0.42) than field capacity $(25 \%)(0.36)$. The interaction of cultivars, boron application as well as various irrigations for leaf area index were presented in Figure 3.CIM-602 had maximum leaf area duration (587.26 days) than CIM-678 (468.22 days) in (Table 3). 
Table 3. Fiber related traits as affected from cultivars, boron application and field capacity

\begin{tabular}{|c|c|c|c|c|c|}
\hline Treatments & $\begin{array}{c}\text { Fiber length } \\
(\mathbf{m m})\end{array}$ & $\begin{array}{c}\text { Fiber } \\
\text { elongation } \\
(\mathbf{m m})\end{array}$ & $\begin{array}{c}\text { Fiber } \\
\text { uniformity } \\
(\mathbf{m m})\end{array}$ & $\begin{array}{c}\text { Fiber } \\
\text { strength } \\
(\mathbf{m m})\end{array}$ & $\begin{array}{c}\text { Micronair } \\
(\boldsymbol{\mu g} / \mathbf{i n c h})\end{array}$ \\
\hline CIM-678 & $20.78 \mathrm{~B}$ & $30.32 \mathrm{~B}$ & $30.32 \mathrm{~B}$ & $15.30 \mathrm{~B}$ & $3.87 \mathrm{~B}$ \\
\hline CIM-602 & $30.56 \mathrm{~A}$ & $30.47 \mathrm{~A}$ & $30.47 \mathrm{~A}$ & $25.22 \mathrm{~A}$ & $4.15 \mathrm{~A}$ \\
\hline Soil application & $22.61 \mathrm{C}$ & $30.38 \mathrm{C}$ & $30.38 \mathrm{C}$ & $18.94 \mathrm{C}$ & $4.00 \mathrm{C}$ \\
\hline Seed treatment & $24.00 \mathrm{~B}$ & $30.40 \mathrm{~B}$ & $30.40 \mathrm{~B}$ & $20.67 \mathrm{~B}$ & $4.01 \mathrm{~B}$ \\
\hline Foliar application & $24.44 \mathrm{~A}$ & $30.41 \mathrm{~A}$ & $30.41 \mathrm{~A}$ & $21.17 \mathrm{~A}$ & $4.03 \mathrm{~A}$ \\
\hline Field capacity (25\%) & $23.17 \mathrm{C}$ & $30.36 \mathrm{C}$ & $30.34 \mathrm{C}$ & $16.89 \mathrm{C}$ & $3.98 \mathrm{C}$ \\
\hline Field capacity (50\%) & $26.11 \mathrm{~B}$ & $30.41 \mathrm{~B}$ & $30.39 \mathrm{~B}$ & $19.67 \mathrm{~B}$ & $4.02 \mathrm{~B}$ \\
\hline Field capacity (75\%) & $27.72 \mathrm{~A}$ & $30.42 \mathrm{~A}$ & $30.40 \mathrm{~A}$ & $21.39 \mathrm{~A}$ & $4.04 \mathrm{~A}$ \\
\hline
\end{tabular}

Application of boron as foliar spray showed higher leaf area duration (528.39 days) than other studied boron application methods. Among irrigation regimes, field capacity $(75 \%)$ showed greater leaf area duration (579.28 days) than field capacity (25\%) (450.11 days). The interactive effect of cultivars, boron use as well as various irrigations for leaf area duration were presented in (Fig. 3). CIM-602 had greater net assimilation rate $\left(1.36 \mathrm{~g} \mathrm{~m}^{-2} \mathrm{day}^{-1}\right)$ than
CIM-678 (1.03 g m ${ }^{-2}$ day $\left.^{-1}\right)$. Foliar application of boron showed maximum net assimilation rate $\left(1.21 \mathrm{~g} \mathrm{~m}^{-2}\right.$ day $\left.^{-1}\right)$ than other studied boron application methods. Among irrigation regimes, field capacity $(75 \%)$ showed greater net assimilation rate (1.22 $\mathrm{g} \mathrm{m}^{-2}$ day $\left.^{-1}\right)$ as compared to field capacity $(25 \%)\left(1.15 \mathrm{~g} \mathrm{~m}^{-2}\right.$ day $\left.^{-1}\right)$. The interaction of cultivars, boron application and irrigation regimes for net assimilation rate were presented in (Fig. 3).

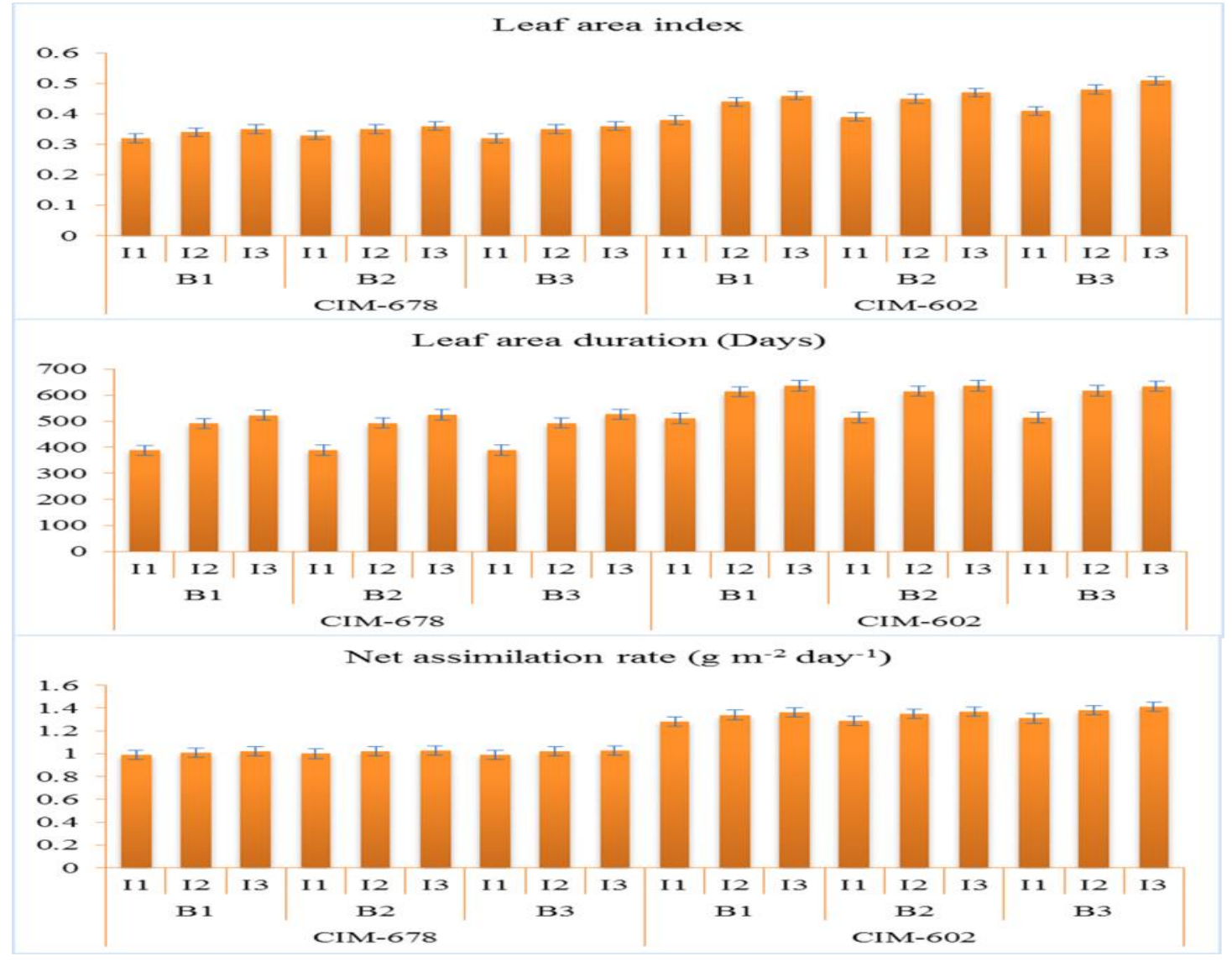

Figure 3. Leaf area index, leaf area duration and net assimilation rate as affected from cultivars, boron application and field capacity 
As regards fiber quality parameters, CIM602 cultivar had higher fiber length (30.56 $\mathrm{mm}$ ) than CIM-678 (20. $78 \mathrm{~mm})$. Among boron application, foliar application showed higher fiber length $(24.44 \mathrm{~mm})$ than other studied boron application methods.

Among irrigation regimes, field capacity $(75 \%)$ showed significant higher fiber length $(27.72 \mathrm{~mm})$ than field capacity $(25 \%)(159.22 \mathrm{~cm})$. The combination of cultivars, boron use and irrigation regimes for fiber length were presented in (Fig. 4). CIM-602 had greater fiber elongation (30.47) than CIM-678 (30.38). Foliar application of boron showed higher fiber elongation $(30.41 \mathrm{~mm})$ than other studied boron application methods. Among irrigation regimes, field capacity $(75 \%)$ showed greater fiber elongation (30.42 $\mathrm{mm})$ than field capacity $(25 \%)(30.36 \mathrm{~mm})$. The interface of cultivars, boron use as well as irrigation regimes for fiber elongation were presented in (Fig. 4). CIM-602 had maximum fiber uniformity $(30.47 \mathrm{~mm})$ than CIM-678 (30.32 mm). Application of boron as foliar showed higher fiber uniformity $(30.47 \mathrm{~mm})$ than other studied boron application methods. Among irrigation regimes, field capacity $(75 \%)$ showed greater fiber uniformity (30.41 $\mathrm{mm})$ than field capacity $(25 \%)(30.38 \mathrm{~mm})$. Interaction of cultivars, boron use as well as irrigation regimes for fiber uniformity were presented in (Fig. 4).

CIM-602 had higher fiber strength (25.22 $\mathrm{mm})$ and micronair (4.15 $\mu \mathrm{g} / \mathrm{inch})$ than CIM-678. Foliar application of boron showed maximum fiber strength $(25.22$ $\mathrm{mm}$ ) and micronair (4.15 $\mu \mathrm{g} / \mathrm{inch})$ than other studied boron application methods. Among irrigation regimes, field capacity (75\%) showed greater fiber strength $(21.39$ $\mathrm{mm})$ and micronair (4.04 $\mu \mathrm{g} / \mathrm{inch})$ than field capacity (25\%). The interaction of cultivars, boron application and irrigation regimes for fiber strength and micron air were presented (Fig. 5). Cost benefit ratio was determined in cotton crop (Table 4).

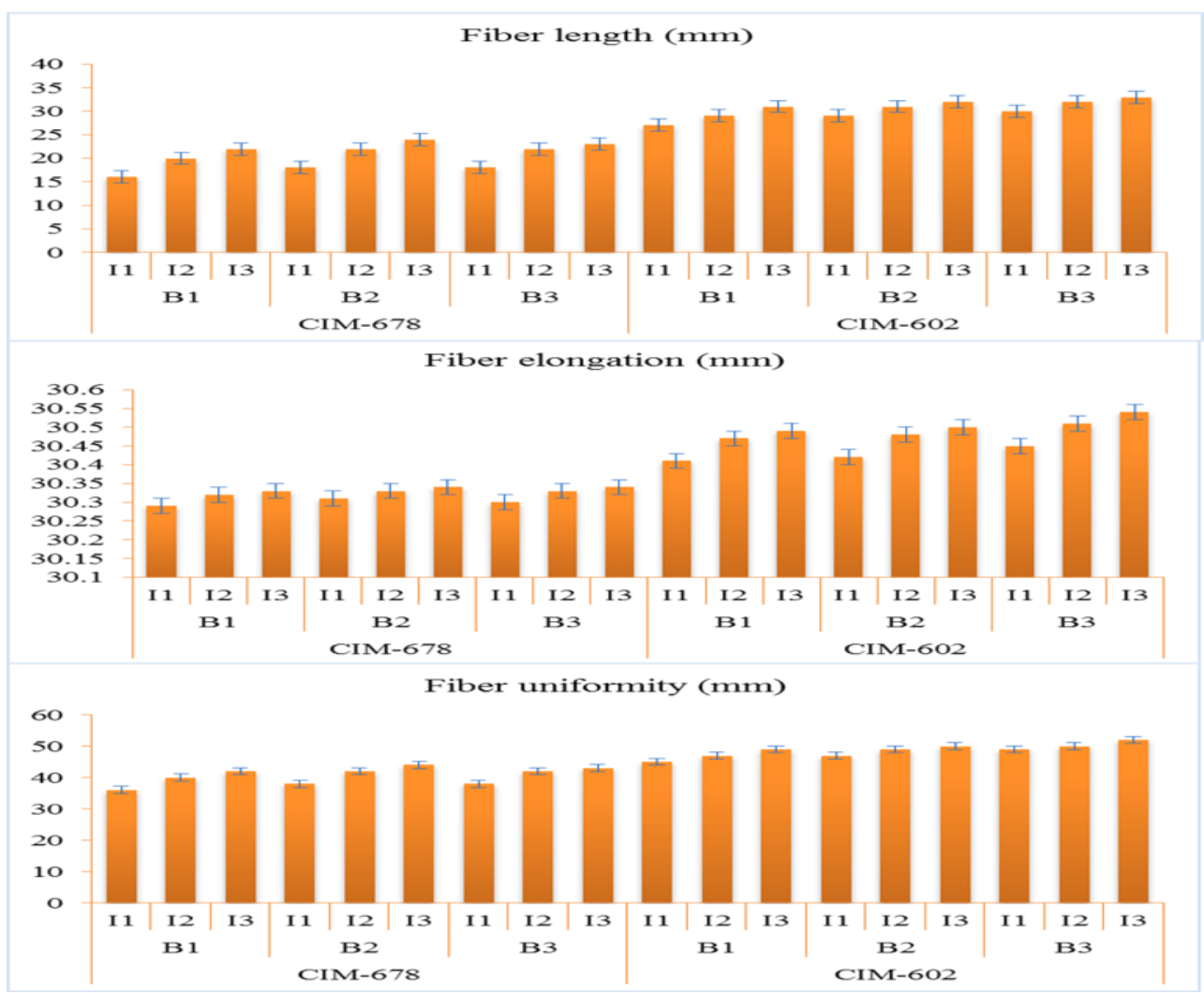

Figure 4. Fiber length, fiber elongation and fiber uniformity as affected from cultivars, boron application and field capacity 


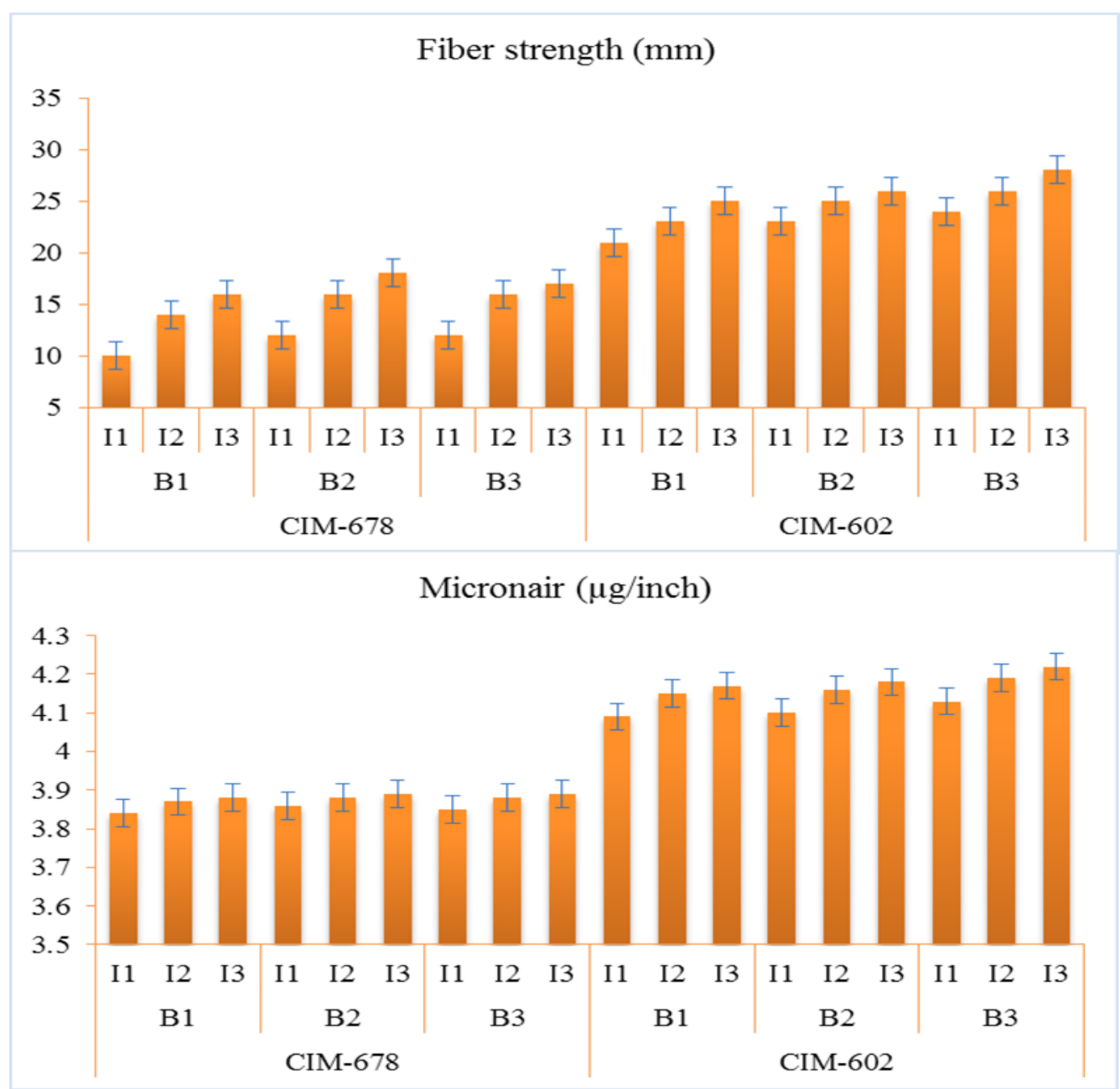

Figure 5. Fiber strength and micronair as affected from cultivars, boron application and field capacity

\section{Discussion}

CIM-678 had maximum growth traits like plant height, boll weight, bolls number/plant, lint yield, seed cotton yield, seed cotton harvest than cultivar CIM-602. Allometry traits such as leaf area index, leaf area duration and net assimilation rate were higher in cultivar CIM-678 than CIM-602. Fiber quality traits i.e. fiber length, elongation, uniformity, strength as well as micronair were greater in cultivar CIM-678 as compared to CIM-602. Between cultivars, CIM-678 had excellent growth, allometry and fiber quality traits. Current study results are under conformity to earlier research work because the cultivars showed variation due to their diverse genetic makeup $[16,17]$.

Among boron application methods, foliar application of boron showed higher plant height, boll weight, bolls number/plant, lint yield, seed cotton yield and seed cotton harvest. Boron spray on leaf enhanced allometry traits comprising leaf area index, leaf area duration and net assimilation rate. Fiber quality parameters containing fiber length, elongation uniformity, strength and micronair were improved in the current experiment. Micronutrients spray on leaf is important source to increase the growth, allometry and fiber quality [18]. Leaf sprays are quickest source of nutrients absorption used for food synthesis because leafs are major factories for food synthesis [19]. Different researcher focused on boron application through and soil and seeds treatment. However, foliar application of boron is very negligible in developing countries especially in Pakistan. 
Table 4. Role of boron application, irrigation regimes and sowing methods on benefit cost ratio (BCR) of cotton

\begin{tabular}{|c|c|c|c|c|c|c|c|}
\hline \multicolumn{8}{|c|}{2015} \\
\hline Treatments & $\begin{array}{c}\text { Yield } \\
\left(\mathrm{kg} \mathrm{ha}^{-1}\right)\end{array}$ & $\begin{array}{c}\text { Value } \\
\left(\text { Rs ha }^{-1}\right)\end{array}$ & $\begin{array}{c}\text { stick value } \\
\left(\text { Rs ha }^{-1}\right)\end{array}$ & $\begin{array}{c}\text { Gross value } \\
\left(\text { Rs ha }^{-1}\right)\end{array}$ & $\begin{array}{l}\text { Total cost } \\
\left(\text { Rs ha }^{-1}\right)\end{array}$ & $\begin{array}{c}\text { Net return } \\
\left(\text { Rs ha }^{-1}\right)\end{array}$ & BCR \\
\hline $\begin{array}{c}\text { Field capacity } \\
(25 \%)\end{array}$ & 2440 & 176900 & 1300 & 178200 & 111950 & 66250 & 1.59 \\
\hline $\begin{array}{c}\text { Field capacity } \\
(50 \%)\end{array}$ & 2570 & 186325 & 1300 & 187625 & 113500 & 74125 & 1.65 \\
\hline $\begin{array}{c}\text { Field capacity } \\
(75 \%)\end{array}$ & 2820 & 204450 & 1300 & 205750 & 110750 & 95000 & 1.86 \\
\hline Flat sowing & 2500 & 181250 & 1200 & 182450 & 111950 & 70500 & 1.63 \\
\hline Ridge sowing & 2630 & 190675 & 1200 & 191875 & 113500 & 78375 & 1.69 \\
\hline Bed sowing & 2880 & 208800 & 1200 & 210000 & 110750 & 99250 & 1.90 \\
\hline \multicolumn{8}{|c|}{2016} \\
\hline $\begin{array}{c}\text { Field capacity } \\
(25 \%)\end{array}$ & 2490 & 180525 & 1200 & 181725 & 111990 & 69735 & 1.62 \\
\hline $\begin{array}{l}\text { Field capacity } \\
(50 \%)\end{array}$ & 2620 & 189950 & 1200 & 191150 & 113510 & 77640 & 1.68 \\
\hline $\begin{array}{l}\text { Field capacity } \\
(75 \%)\end{array}$ & 2870 & 208075 & 1200 & 209275 & 110770 & 98505 & 1.89 \\
\hline Flat sowing & 2550 & 184875 & 1200 & 186075 & 111990 & 74085 & 1.66 \\
\hline Ridge sowing & 2680 & 194300 & 1200 & 195500 & 113510 & 81990 & 1.72 \\
\hline Bed sowing & 2930 & 212425 & 1200 & 213625 & 110770 & 102855 & 1.93 \\
\hline
\end{tabular}

Regarding irrigation regimes, plant growth parameters were higher under field capacity $(75 \%)$. Field capacity $(75 \%)$ enhanced allometry traits comprising leaf area index, leaf area duration and net assimilation rate. Fiber quality parameters having fiber length, fiber elongation, fiber uniformity, fiber strength and micronair were improved under field capacity (75\%). Different irrigation regimes have potential to protect huge losses in form of heavy irrigation. Present results are in line to previous researches [20]. Different researcher focused on different irrigation regimes [21]. The combination of cultivars, boron application and different irrigation regimes were showed greater improvement in cotton crop. Current study is line to previous research work [22].

\section{Conclusion}

Cotton is major cash crop globally and important for foreign exchange. In the light of present observations, CIM-678 had excellent growth, allometry and fiber traits than CIM-602. Foliar application method is more successful for cotton crop than soil and seeds treatment. Field capacity (75\%) resulting in higher growth, allometry and fiber quality traits than other irrigation regimes. It has been concluded that cotton crop can be improved by spraying boron on leaf and selecting drought resistant cultivars. Moreover, application of different molecular approaches may be effective for increase of development of tolerant cultivars.

\section{Authors' contributions}

Conceived and designed the experiments: $\mathrm{H}$ Ali, Performed the experiments: A Ahmad, Analyzed the data: R Ahmad, Contributed materials/ analysis/ tools: $\mathrm{H}$ Ali \& M Irfan, Wrote the paper: W Hassan \& S Ahmad.

\section{Acknowledgements}

The authors are very thankful to the Central Cotton Research Institute, Multan for providing the plant material and experimental site and Bahauddin Zakariya University, Multan for the financial support to carry out this work.

\section{References}

1. Ali H, Ahmad A \& Hussain S (2020). The effect of exogenous phosphorous application on growth, yield, quality and net returns of upland cotton (Gossipium hirsutum L.). Appl Ecol 
Environ Res 18: 769-781.

2. Ali H, Afzal MN \& Muhammad D (2009). Effect of sowing dates and plant spacing on growth and dry matter partitioning in cotton (Gossypium hirsutum L.). Pak J Bot 41: 2145-2155.

3. Ali H, Muhammad D \& Abid SA (2005). Weed control practices in cotton (Gossypium hirsutum L.) planted on bed and furrow. Pak J Weed Sci Res 11: 43-48.

4. Amouzou KA, Naab JB, Lamers JP, Borgemeister C, Becker M \& Vlek PL (2018). CROPGRO-Cotton model for determining climate change impacts on yield, water-and N-use efficiencies of cotton in the Dry Savanna of West Africa. Agric Syst 165: 85-96.

5. Ali H, Afzal MN, Ahmad F, Ahmad S, Akhtar M \& Atif R (2011). Effect of sowing dates, plant spacing and nitrogen application on growth and productivity on cotton crop. Int $J$ Sci Engin Res 2: 1-6.

6. Ali H \& Hameed RA (2011). Growth, yield and yield components of American cotton (Gossypium hirsutum L.) as affected by cultivars and nitrogen fertilizer. Chem Anal 15: 30.

7. Blaise D, Singh JV, Bonde AN, Tekale KU \& Mayee CD (2005). Effects of farmyard manure and fertilizers on yield, fibre quality and nutrient balance of rainfed cotton (Gossypium hirsutum). Bio-res Technol 96: 345349.

8. Ali H, Iqbal N, Shahzad AN, Sarwar N, Ahmad S \& Mehmood A (2013). Seed priming improves irrigation water use efficiency, yield, and yield components of late-sown wheat under limited water conditions. Turk J Agric Forest 37: 534-544.

9. Mahpara S, Shahnawaz M, Rehman K, Ahmad R \& Khan FU (2019). Nitrogen fertilization induced drought tolerance in sunflower: a review. Pure Appl Biol 8: 1675-1683.

10. Rochester IJ (2007). Nutrient uptake and export from an Australian cotton field. Nutr Cyc Agroecosysts 77(3): 213-123.

11. Irfan M, Ali H, Ahmad S, Sattar A, Areeb A, Hussain S, Shabbir RN, Sarwar N \& Hussain F (2017). Various tillage systems and sowing methods affect growth and yield related characters of cotton. J Arab Crop Market 1: 09-19.

12. Yu C, Wang $X, H u$ B, Yang C, Sui N, Liu R, Meng Y \& Zhou Z (2016). Effects of wheat straw incorporation in cotton-wheat double cropping system on nutrient status and growth in cotton. Field Crops Res 197: 39-51.

13. Baio FHR, Gabriel RRF, Zanin ARA, Campos CNS, Roque CG \& Teodoro PE (2020). Application technology of boron via foliar and its effects on cotton crop phenology. Brazil J Develop 6: 7367-7379.

14. Ali H, Afzal MN, Ahmad S, Muhammad D, Hasnain Z, Perveen R \& Kazmi MH (2010). Quantitative and qualitative traits of Gossypium hirsutum L. as affected by agronomic practices. $J$ Food Agric Environ 8: 945-948.

15. Upadhyaya H \& Panda SK (2019). Drought Stress Responses and Its Management in Rice. In Advances in Rice Research for Abiotic Stress Tolerance. Woodhead Publishing, 177200.

16. Jayakumar M, Surendran U \& Manickasundaram P (2014). Drip fertigation effects on yield, nutrient uptake and soil fertility of Bt Cotton in semi-arid tropics. Int J Plant Product 8: 375-90.

17. Chen Z, Khan A, Shi X, Hao X, Tan DKY \& Luo H (2020). Water-nutrient management enhances root morphophysiological functioning, phosphorus absorption, transportation and utilization of cotton in arid region. Ind Crop Prod 143: 111975.

18. Emam S (2020). Estimation of straw, seed and oil yields for flax plants (Linum usitatissimum L.) cultivars of foliar application of $\mathrm{Mn}, \mathrm{Fe}$ and $\mathrm{Zn}$ 
under dry environment. Egy J Agron 11: 112-117.

19. Ziogas V, Michailidis M, Karagiannis E, Tanou G \& Molassiotis A (2020). Manipulating fruit quality through foliar nutrition. In Fruit Crops Elsevier pp. 401-417.

20. Alinejad S, Sarabi V, Bakhtvari ARS \& Hashempour H (2020). Variation in physiological traits, yield and secondary metabolites of jimsonweed (Datura stramonium L.) under different irrigation regimes and nutrition systems. Indus Crop Prod 143: 111916.
21. Hussain M, Tariq AF, Nawaz A, Nawaz M, Sattar A, Ul-Allah S \& Wakeel A (2020). Efficacy of fertilizing method for different potash sources in cotton (Gossypium hirsutum L.) nutrition under arid climatic conditions. PLoS One 15: e0228335.

22. Li M, Xiao J, Bai Y, Du Y, Zhang F, Cheng H \& Wang H (2020). Response mechanism of cotton growth to water and nutrients under drip irrigation with plastic mulch in Southern Xinjiang. $J$ Sensor 11-125. 